\title{
Article
}

\section{On the vector Fourier multipliers for compact groups}

\author{
Abudulaï Issa ${ }^{1}$ and Yaogan Mensah ${ }^{1,2, *}$ \\ 1 Department of Mathematics, University of Lomé, POBox 1515, Lomé, Togo. issaabudulai13@gmail.com \\ 2 International Chair in Mathematical Physics and Applications (ICMPA)-Unesco Chair, University of \\ Abomey-Calavi, Benin. \\ * Correspondence: mensahyaogan2@gmail.com
}

Received: 29 August 2019; Accepted: 17 November 2019; Published: 7 December 2019.

\begin{abstract}
This paper studies some properties of the Fourier multiplier operators on a compact group when the underlying multiplication functions (the symbols) defined on the dual object take values in a Banach algebra. More precisely, boundedness properties for such Fourier multiplier operators for the space of Bochner strong integrable functions and for the (vector) $p$-Fourier spaces are investigated.
\end{abstract}

Keywords: Compact group, Fourier transform, Fourier multiplier, $p$-Fourier space.

MSC: 43A22, 43A30, 43A15.

\section{Introduction}

$\mathbf{T}$ he theory of Fourier multipliers is part of the theory of Fourier integral operators and localization operators. Roughly speaking, a Fourier multiplier is an operator defined through a multiplication by a symbol on a function's frequency spectrum. It is a way to reshape the frequencies involved in the function. Therefore this theory has many applications for instance in Signal processing where the Fourier multiplier is called a filter. Research on the Fourier multipliers is very active and quite flourishing. As recent articles in this field we can quote [1-3].

In [4], Atto et al. investigated the Fourier multipliers for a kind of $p$-Fourier spaces introduced in [5]. They obtained important results related to the boundedness of such operators. However, the underlying multiplication function (the symbol) takes values in the set of complex numbers though authors dealt with the Fourier transform of vector valued functions. It may have been interesting to consider vector valued symbols. So, in order to harmonize things, it seems necessary to complete/extend the study by the case where the symbols are vector valued functions. This is the main purpose of this paper. Thanks to the Fourier inversion formula in [6], it is possible to introduce what we call a vector Fourier multiplier.

The paper is organized as follows. In Section 2, we set some preliminaries related to the Fourier transform of vector valued functions. In Section 3, we investigate properties of the Fourier multipliers for Bochner integrable functions and in Section 4 , we study the Fourier multipliers for $p$-Fourier spaces.

\section{Preliminaries}

Details on group representations can be found in $[7,8]$. Let $G$ be a compact group with normalized Haar measure $d x$. We denote by $\widehat{G}$ the unitary dual of $G$, that is the set of equivalence classes of unitary irreducible representations of $G$. In each class $\sigma \in \widehat{G}$, we choose an element, still denoted $\sigma$, with representation space $H_{\sigma}$ the dimension of which is denoted by $d_{\sigma}$. We designate by $\left(\xi_{1}^{\sigma}, \xi_{2}^{\sigma}, \ldots . ., \xi_{d_{\sigma}}^{\sigma}\right)$ a basis of $H_{\sigma}$. Let $\mathfrak{A}$ be a complex Banach algebra. The Fourier transform $\widehat{f}$ of a strong Bochner integrable function $f \in L^{1}(G, \mathfrak{A})$ is given by the formula

$$
\widehat{f}(\sigma)(\xi \otimes \eta)=\int_{G}\left\langle\sigma\left(x^{-1}\right) \xi, \eta\right\rangle f(x) d x
$$

where $\langle\cdot, \cdot\rangle$ denotes the inner product in $H_{\sigma}$. Here $\widehat{f}(\sigma)$ is a bounded linear operator from $H_{\sigma} \otimes \bar{H}_{\sigma}$ into $\mathfrak{A}$ where $\bar{H}_{\sigma}$ is the conjugate Hilbert space of $H_{\sigma}$; see [9]. Now set 


$$
u_{i j}^{\sigma}(x)=\left\langle\sigma(x) \xi_{j}^{\sigma}, \xi_{i}^{\sigma}\right\rangle
$$

The functions $u_{i j}^{\sigma}$ satisfy the Schur orthogonality relations

$$
\int_{G} u_{i j}^{\sigma}(x) \overline{u_{m n}^{\sigma^{\prime}}(x)} d x=\frac{1}{d_{\sigma}} \delta_{i m} \delta_{j n} \delta_{\sigma \sigma^{\prime}}
$$

where $\delta$ is the Kronecker delta symbol.

The Fourier inversion formula is

$$
f=\sum_{\sigma \in \widehat{G}} d_{\sigma} \sum_{i=1}^{d_{\sigma}} \sum_{j=1}^{d_{\sigma}} \widehat{f}(\sigma)\left(\xi_{j}^{\sigma} \otimes \xi_{i}^{\sigma}\right) u_{i j}^{\sigma} .
$$

Using this inversion formula, we can define what are vector Fourier multipliers and tackle the study of their properties. This is done in the next two sections.

\section{Vector Fourier multipliers for $L^{1}(G, \mathfrak{A})$}

Set

$$
\mathcal{L}(\widehat{G}, \mathfrak{A})=\prod_{\sigma \in \widehat{G}} \mathcal{L}\left(H_{\sigma} \otimes \bar{H}_{\sigma}, \mathfrak{A}\right)
$$

where $\mathcal{L}\left(H_{\sigma} \otimes \bar{H}_{\sigma}, \mathfrak{A}\right)$ designates the set of linear operators from $H_{\sigma} \otimes \bar{H}_{\sigma}$ into $\mathfrak{A}$.

Following [6], we consider the product $\times$ on $\mathcal{L}(\widehat{G}, \mathfrak{A})$ defined as follows. If $\phi_{1}, \phi_{2} \in \mathcal{L}(\widehat{G}, \mathfrak{A})$ then $\phi_{1} \times \phi_{2}$ is given by

$$
\left(\phi_{1} \times \phi_{2}\right)(\sigma)\left(\xi_{j}^{\sigma} \otimes \xi_{i}^{\sigma}\right)=\sum_{k=1}^{d_{\sigma}} \phi_{1}(\sigma)\left(\xi_{k}^{\sigma} \otimes \xi_{i}^{\sigma}\right) \phi_{2}(\sigma)\left(\xi_{j}^{\sigma} \otimes \xi_{k}^{\sigma}\right)
$$

Let $\varphi: \widehat{G} \rightarrow \mathfrak{A}$ be a bounded function. We define the vector Fourier multiplier $T_{\varphi}$ by the formula

$$
T_{\varphi} f=\sum_{\sigma \in \widehat{G}} d_{\sigma} \sum_{i=1}^{d_{\sigma}} \sum_{j=1}^{d_{\sigma}} \varphi(\sigma) \widehat{f}(\sigma)\left(\xi_{j}^{\sigma} \otimes \xi_{i}^{\sigma}\right) u_{i j}^{\sigma} .
$$

What we consider here generalizes the case treated in [4] which corresponds to the particular case $\mathfrak{A}=\mathbb{C}$.

We denote by $\mathcal{M}\left(L^{1}(G, \mathfrak{A})\right)$ the set of all vector Fourier multipliers on $L^{1}(G, \mathfrak{A})$. We introduce the following operation which we may need.

$$
(\varphi \otimes \widehat{f})(\sigma)(\xi \otimes \eta)=\varphi(\sigma) \widehat{f}(\sigma)(\xi \otimes \eta), \xi \in H_{\sigma}, \eta \in \bar{H}_{\sigma} .
$$

Taking inspiration from [6, Therem 3.1.] we state the following theorem.

Theorem 1. If $f, g \in L^{1}(G, \mathfrak{A})$ then $\widehat{f * g}=\widehat{f} \times \widehat{g}$.

The next theorem gives a characterization of the vector Fourier multipliers on $L^{1}(G, \mathfrak{A})$.

\section{Theorem 2.}

$$
T_{\varphi} \in \mathcal{M}\left(L^{1}(G, \mathfrak{A})\right) \Longleftrightarrow \widehat{T_{\varphi} f}=\varphi \otimes \widehat{f}, \forall f \in L^{1}(G, \mathfrak{A})
$$


Proof. Let $T_{\varphi} \in \mathcal{M}\left(L^{1}(G, \mathfrak{A})\right)$. Let $\sigma^{\prime} \in \widehat{G}$. Vectors $\xi \in H_{\sigma^{\prime}}$ and $\eta \in \bar{H}_{\sigma^{\prime}}$ can be written in the forms $\xi=$ $\sum_{n=1}^{d_{\sigma^{\prime}}} \alpha_{n} \xi_{n}^{\sigma^{\prime}}$ and $\eta=\sum_{m=1}^{d_{\sigma^{\prime}}} \bar{\beta}_{m} \xi_{m}^{\sigma^{\prime}}$ in the basis $\left(\xi_{1}^{\sigma^{\prime}}, \xi_{2}^{\sigma^{\prime}}, \ldots, \xi_{\sigma_{\sigma^{\prime}}}^{\sigma^{\prime}}\right)$ of $H_{\sigma^{\prime}}$. Then

$$
\begin{aligned}
\widehat{T_{\varphi} f}\left(\sigma^{\prime}\right)(\xi \otimes \eta) & =\widehat{T_{\varphi} f}\left(\sigma^{\prime}\right)\left(\sum_{n=1}^{d_{\sigma^{\prime}}} \alpha_{n} \xi_{n}^{\sigma^{\prime}} \otimes \sum_{m=1}^{d_{\sigma^{\prime}}} \bar{\beta}_{m} \xi_{m}^{\sigma^{\prime}}\right) \\
& =\sum_{n=1}^{d_{\sigma^{\prime}}} \sum_{m=1}^{d_{\sigma^{\prime}}} \alpha_{n} \bar{\beta}_{m} \widehat{T_{\varphi} f}\left(\sigma^{\prime}\right)\left(\xi_{n}^{\sigma^{\prime}} \otimes \xi_{m}^{\sigma^{\prime}}\right) \\
& =\sum_{n=1}^{d_{\sigma^{\prime}}} \sum_{m=1}^{d_{\sigma^{\prime}}} \alpha_{n} \bar{\beta}_{m} \int_{G}\left\langle\sigma^{\prime}\left(x^{-1}\right) \xi_{n}^{\sigma^{\prime}}, \xi_{m}^{\sigma^{\prime}}\right\rangle\left(T_{\varphi} f\right)(x) d x \\
& =\sum_{n=1}^{d_{\sigma^{\prime}}} \sum_{m=1}^{d_{\sigma^{\prime}}} \alpha_{n} \bar{\beta}_{m} \int_{G} \overline{u_{n m}^{\sigma^{\prime}}(x)}\left(T_{\varphi} f\right)(x) d x \\
& =\sum_{n=1}^{d_{\sigma^{\prime}}} \sum_{m=1}^{d_{\sigma^{\prime}}} \alpha_{n} \bar{\beta}_{m} \int_{G} \overline{u_{n m}^{\sigma^{\prime}}(x)} \sum_{\sigma \in \widehat{G}} d_{\sigma} \sum_{i=1}^{d_{\sigma}} \sum_{j=1}^{d_{\sigma}} \varphi(\sigma) \widehat{f}(\sigma)\left(\xi_{j}^{\sigma} \otimes \xi_{i}^{\sigma}\right) u_{i j}^{\sigma}(x) d x \\
& =\sum_{n=1}^{d_{\sigma^{\prime}}} \sum_{m=1}^{d_{\sigma^{\prime}}} \alpha_{n} \bar{\beta}_{m} \sum_{\sigma \in \widehat{G}} d_{\sigma} \sum_{i=1}^{d_{\sigma}} \sum_{j=1}^{d_{\sigma}} \varphi(\sigma) \widehat{f}(\sigma)\left(\xi_{j}^{\sigma} \otimes \xi_{i}^{\sigma}\right) \int_{G} \overline{u_{m n}^{\sigma^{\prime}}(x)} u_{i j}^{\sigma}(x) d x .
\end{aligned}
$$

By appealing to the Schur orthogonality relations, we get

$$
\begin{aligned}
\widehat{T_{\varphi} f}\left(\sigma^{\prime}\right)(\xi \otimes \eta) & =\sum_{n=1}^{d_{\sigma^{\prime}}} \sum_{m=1}^{d_{\sigma^{\prime}}} \alpha_{n} \bar{\beta}_{m} d_{\sigma^{\prime}} \sum_{i=1}^{d_{\sigma}} \sum_{j=1}^{d_{\sigma}} \varphi\left(\sigma^{\prime}\right) \widehat{f}\left(\sigma^{\prime}\right)\left(\xi_{j}^{\sigma^{\prime}} \otimes \xi_{i}^{\sigma^{\prime}}\right)\left(\frac{1}{d_{\sigma^{\prime}}} \delta_{i m} \delta_{j n}\right) \\
& =\sum_{n=1}^{d_{\sigma^{\prime}}} \sum_{m=1}^{d_{\sigma^{\prime}}} \alpha_{n} \bar{\beta}_{m} \varphi\left(\sigma^{\prime}\right) \widehat{f}\left(\sigma^{\prime}\right)\left(\xi_{n}^{\sigma^{\prime}} \otimes \xi_{m}^{\sigma^{\prime}}\right) \\
& =\varphi\left(\sigma^{\prime}\right) \widehat{f}\left(\sigma^{\prime}\right)\left(\sum_{n=1}^{d_{\sigma^{\prime}}} \alpha_{n} \xi_{n}^{\sigma^{\prime}} \otimes \sum_{m=1}^{d_{\sigma^{\prime}}} \overline{\beta_{m}} \xi_{m}^{\sigma^{\prime}}\right) \\
& =\varphi\left(\sigma^{\prime}\right) \widehat{f}\left(\sigma^{\prime}\right)(\xi \otimes \eta) \\
& =(\varphi \otimes \widehat{f})\left(\sigma^{\prime}\right)(\xi \otimes \eta)
\end{aligned}
$$

Thus $\widehat{T_{\varphi} f}=\varphi \otimes \widehat{f}$.

Conversely, let us assume that $\forall f \in L^{1}(G, \mathfrak{A}), \widehat{T_{\varphi} f}=\varphi \otimes \widehat{f}$. Then, using the inversion formula we obtain

$$
\begin{aligned}
T_{\varphi} f & =\sum_{\sigma \in \widehat{G}} d_{\sigma} \sum_{i=1}^{d_{\sigma}} \sum_{j=1}^{d_{\sigma}} \widehat{T_{\varphi} f}(\sigma)\left(\xi_{j}^{\sigma} \otimes \xi_{i}^{\sigma}\right) u_{i j}^{\sigma} \\
& =\sum_{\sigma \in \widehat{G}} d_{\sigma} \sum_{i=1}^{d_{\sigma}} \sum_{j=1}^{d_{\sigma}}(\varphi \otimes \widehat{f})(\sigma)\left(\xi_{j}^{\sigma} \otimes \xi_{i}^{\sigma}\right) u_{i j}^{\sigma} \\
& =\sum_{\sigma \in \widehat{G}} d_{\sigma} \sum_{i=1}^{d_{\sigma}} \sum_{j=1}^{d_{\sigma}} \varphi(\sigma) \widehat{f}(\sigma)\left(\xi_{j}^{\sigma} \otimes \xi_{i}^{\sigma}\right) u_{i j}^{\sigma} .
\end{aligned}
$$

Thus $T_{\varphi} f$ is a vector Fourier multiplier for $L^{1}(G, \mathfrak{A})$.

Theorem 3. If $T_{\varphi}, T_{\phi} \in \mathcal{M}\left(L^{1}(G, \mathfrak{A})\right)$ and $f, g \in L^{1}(G, \mathfrak{A})$ then the following equalities hold:

$$
\begin{gathered}
T_{\varphi}(f * g)=T_{\varphi} f * g . \\
T_{\varphi \phi}(f * g)=\left(T_{\varphi} T_{\phi} f\right) * g .
\end{gathered}
$$


Proof. Let $T_{\varphi} \in \mathcal{M}\left(L^{1}(G, \mathfrak{A})\right)$ and $f, g \in L^{1}(G, \mathfrak{A})$.

$$
\begin{aligned}
\mathcal{F}\left(T_{\varphi}(f * g)\right)(\sigma)\left(\xi_{i}^{\sigma} \otimes \xi_{j}^{\sigma}\right) & =(\varphi \otimes \widehat{f * g})(\sigma)\left(\xi_{i}^{\sigma} \otimes \xi_{j}^{\sigma}\right) \\
& =\varphi(\sigma) \widehat{f * g}(\sigma)\left(\xi_{i}^{\sigma} \otimes \xi_{j}^{\sigma}\right) \\
& =\varphi(\sigma)\left[(\widehat{f} \times \widehat{g})(\sigma)\left(\xi_{i}^{\sigma} \otimes \xi_{j}^{\sigma}\right)\right] \\
& =\varphi(\sigma)\left[\sum_{k=1}^{d_{\sigma}} \widehat{f}(\sigma)\left(\xi_{k}^{\sigma} \otimes \xi_{j}^{\sigma}\right) \widehat{g}(\sigma)\left(\xi_{i}^{\sigma} \otimes \xi_{k}^{\sigma}\right)\right] \\
& =\sum_{k=1}^{d_{\sigma}} \varphi(\sigma) \widehat{f}(\sigma)\left(\xi_{k}^{\sigma} \otimes \xi_{j}^{\sigma}\right) \widehat{g}(\sigma)\left(\xi_{i}^{\sigma} \otimes \xi_{k}^{\sigma}\right) \\
& =\sum_{k=1}^{d_{\sigma}}\left[\varphi(\sigma) \widehat{f}(\sigma)\left(\xi_{k}^{\sigma} \otimes \xi_{j}^{\sigma}\right)\right] \widehat{g}(\sigma)\left(\xi_{i}^{\sigma} \otimes \xi_{k}^{\sigma}\right) \\
& =\sum_{k=1}^{d_{\sigma}}\left[(\varphi \otimes \widehat{f})(\sigma)\left(\xi_{k}^{\sigma} \otimes \xi_{j}^{\sigma}\right)\right] \widehat{g}(\sigma)\left(\xi_{i}^{\sigma} \otimes \xi_{k}^{\sigma}\right) \\
& \left.=\sum_{k=1}^{d_{\sigma}}\left[\widehat{T_{\varphi} f}\right)(\sigma)\left(\xi_{k}^{\sigma} \otimes \xi_{j}^{\sigma}\right)\right] \widehat{g}(\sigma)\left(\xi_{i}^{\sigma} \otimes \xi_{k}^{\sigma}\right) \\
& =\left(\widehat{T_{\varphi} f} \times \widehat{g}\right)(\sigma)\left(\xi_{i}^{\sigma} \otimes \xi_{j}^{\sigma}\right) \\
& =\mathcal{F}\left(T_{\varphi} f * g\right)(\sigma)\left(\xi_{i}^{\sigma} \otimes \xi_{j}^{\sigma}\right)
\end{aligned}
$$

Since the Fourier transformation is injective, we have $T_{\varphi}(f * g)=T_{\varphi} f * g$. Let $T_{\varphi}, T_{\phi} \in \mathcal{M}\left(L^{1}(G, \mathfrak{A})\right)$ and $f, g \in L^{1}(G, \mathfrak{A})$.

$$
\begin{aligned}
\mathcal{F}\left(T_{\varphi \phi}(f * g)\right)(\sigma)\left(\xi_{i}^{\sigma} \otimes \xi_{j}^{\sigma}\right) & =(\varphi \phi \otimes \widehat{f * g})(\sigma)\left(\xi_{i}^{\sigma} \otimes \xi_{j}^{\sigma}\right) \\
& =(\varphi \phi)(\sigma)(\widehat{f * g})(\sigma)\left(\xi_{i}^{\sigma} \otimes \xi_{j}^{\sigma}\right) \\
& =\varphi(\sigma) \phi(\sigma)(\widehat{f} \times \widehat{g})(\sigma)\left(\xi_{i}^{\sigma} \otimes \xi_{j}^{\sigma}\right) \\
& =\varphi(\sigma) \phi(\sigma) \sum_{k=1}^{d_{\sigma}} \widehat{f}(\sigma)\left(\xi_{k}^{\sigma} \otimes \xi_{j}^{\sigma}\right) \widehat{g}(\sigma)\left(\xi_{i}^{\sigma} \otimes \xi_{k}^{\sigma}\right) \\
& =\varphi(\sigma) \sum_{k=1}^{d_{\sigma}} \phi(\sigma) \widehat{f}(\sigma)\left(\xi_{k}^{\sigma} \otimes \xi_{j}^{\sigma}\right) \widehat{g}(\sigma)\left(\xi_{i}^{\sigma} \otimes \xi_{k}^{\sigma}\right) \\
& =\varphi(\sigma) \sum_{k=1}^{d_{\sigma}}(\phi \otimes \widehat{f})(\sigma)\left(\xi_{k}^{\sigma} \otimes \xi_{j}^{\sigma}\right) \widehat{g}(\sigma)\left(\xi_{i}^{\sigma} \otimes \xi_{k}^{\sigma}\right) \\
& =\varphi(\sigma) \sum_{k=1}^{d_{\sigma}} \widehat{T_{\phi} f}(\sigma)\left(\xi_{k}^{\sigma} \otimes \xi_{j}^{\sigma}\right) \widehat{g}(\sigma)\left(\xi_{i}^{\sigma} \otimes \xi_{k}^{\sigma}\right) \\
& =\sum_{k=1}^{d_{\sigma}} \varphi(\sigma) \widehat{T_{\phi} f}(\sigma)\left(\xi_{k}^{\sigma} \otimes \xi_{j}^{\sigma}\right) \widehat{g}(\sigma)\left(\xi_{i}^{\sigma} \otimes \xi_{k}^{\sigma}\right) \\
& =\sum_{k=1}^{d_{\sigma}}\left(\varphi \otimes \widehat{T_{\phi} f}\right)(\sigma)\left(\xi_{k}^{\sigma} \otimes \xi_{j}^{\sigma}\right) \widehat{g}(\sigma)\left(\xi_{i}^{\sigma} \otimes \xi_{k}^{\sigma}\right) \\
& =\sum_{k=1}^{d_{\sigma}} \widehat{T_{\varphi} T_{\phi} f}(\sigma)\left(\xi_{k}^{\sigma} \otimes \xi_{j}^{\sigma}\right) \widehat{g}(\sigma)\left(\xi_{i}^{\sigma} \otimes \xi_{k}^{\sigma}\right) \\
& =\left(\widehat{T_{\varphi} T_{\phi} f} \times \widehat{g}\right)(\sigma)\left(\xi_{i}^{\sigma} \otimes \xi_{j}^{\sigma}\right) \\
& =\mathcal{F}\left(\left(T_{\varphi} T_{\phi} f\right) * g\right)(\sigma)\left(\xi_{i}^{\sigma} \otimes \xi_{j}^{\sigma}\right) .
\end{aligned}
$$

Therefore, by the injectivity of the Fourier transformation, we have

$$
T_{\varphi \phi}(f * g)=\left(T_{\varphi} T_{\phi} f\right) * g .
$$


For $\psi \in \mathcal{L}(\widehat{G}, \mathfrak{A})$, we set

$$
\|\psi\|_{\infty}=\sup \{\|\psi(\sigma)\|: \sigma \in \widehat{G}\}
$$

where

$$
\|\psi(\sigma)\|=\sup \{\|\psi(\sigma)(\xi \otimes \eta)\|:\|\xi\| \leq 1,\|\eta\| \leq 1\} .
$$

We also consider the set

$$
\mathcal{L}_{\infty}(\widehat{G}, \mathfrak{A})=\left\{\psi \in \mathcal{L}(\widehat{G}, \mathfrak{A}):\|\psi\|_{\infty}<\infty\right\}
$$

We can now state the following theorem.

Theorem 4. If $T_{\varphi} \in \mathcal{M}\left(L^{1}(G, \mathfrak{A})\right)$ and $f \in L^{1}(G, \mathfrak{A})$ then $\widehat{T_{\varphi} f} \in \mathcal{L}_{\infty}(\widehat{G}, \mathfrak{A})$ and

$$
\left\|\widehat{T_{\varphi} f}\right\|_{\infty} \leq\|\varphi\|_{\infty}\|f\|_{1} \text {. }
$$

Proof. Let $\xi \otimes \eta \in H_{\sigma} \otimes \bar{H}_{\sigma}$.

$$
\begin{aligned}
\left\|\widehat{T_{\varphi} f}(\sigma)(\xi \otimes \eta)\right\| & =\|(\varphi \otimes \widehat{f})(\sigma)(\xi \otimes \eta)\| \\
& =\|\varphi(\sigma) \widehat{f}(\sigma)(\xi \otimes \eta)\| \\
& \leq\|\varphi(\sigma)\| \widehat{f}(\sigma)(\xi \otimes \eta) \| \\
& \leq\|\varphi\|_{\infty}\|\xi\|\|\eta\|\|f\|_{1} .
\end{aligned}
$$

Hence

$$
\left\|\widehat{T_{\varphi} f}(\sigma)\right\| \leq\|\varphi\|_{\infty}\|f\|_{1}, \forall \sigma \in \widehat{G}
$$

Thus

$$
\left\|\widehat{T_{\varphi} f}\right\|_{\infty} \leq\|\varphi\|_{\infty}\|f\|_{1}
$$

\section{Vector Fourier multipliers on $p$-Fourier spaces}

For $1 \leq p<\infty$, consider

$$
\mathcal{L}_{p}(\widehat{G}, \mathfrak{A})=\left\{\psi \in \mathcal{L}(\widehat{G}, \mathfrak{A}): \sum_{\sigma \in \widehat{G}} d_{\sigma} \sum_{i=1}^{d_{\sigma}} \sum_{j=1}^{d_{\sigma}}\left\|\psi(\sigma)\left(\xi_{i}^{\sigma} \otimes \xi_{j}^{\sigma}\right)\right\|^{p}<\infty\right\} .
$$

The space $\mathcal{L}_{p}(\widehat{G}, \mathfrak{A})$ is a Banach space if it is endowed with the norm

$$
\|\psi\|_{\mathcal{L}_{p}}=\left(\sum_{\sigma \in \widehat{G}} d_{\sigma} \sum_{i=1}^{d_{\sigma}} \sum_{j=1}^{d_{\sigma}}\left\|\psi(\sigma)\left(\xi_{i}^{\sigma} \otimes \xi_{j}^{\sigma}\right)\right\|^{p}\right)^{\frac{1}{p}}
$$

Call $p$-Fourier spaces the spaces

$$
\mathcal{A}_{p}(G, \mathfrak{A})=\left\{f \in L^{1}(G, \mathfrak{A}): \widehat{f} \in \mathcal{L}_{p}(\widehat{G}, \mathfrak{A})\right\}, 1 \leq p<\infty .
$$

On the space $\mathcal{A}_{p}(G, \mathfrak{A})$ the following two norms are defined:

$$
\|f\|_{\mathcal{A}_{p}}=\|f\|_{1}+\|\widehat{f}\|_{\mathcal{L}_{p}}, \quad f \in \mathcal{A}_{p}(G, \mathfrak{A})
$$




$$
\|f\|^{\mathcal{A}_{p}}=\|\widehat{f}\|_{\mathcal{L}_{p},} \quad f \in \mathcal{A}_{p}(G, \mathfrak{A})
$$

where $\|.\|_{1}$ and $\|\cdot\|_{\mathcal{L}_{p}}$ denote the norm in $L^{1}(G, \mathfrak{A})$ and the norm in $\mathcal{L}_{p}(\widehat{G}, \mathfrak{A})$ respectively. Equipped with each of the two norms $\|\cdot\|_{\mathcal{A}_{p}}$ and $\|.\| \mathcal{A}_{p}$, it had been proved that the space $\mathcal{A}_{p}(G, \mathfrak{A})$ is a Banach space [5].

The following theorem expresses the invariance of the space $\mathcal{A}_{p}(G, \mathfrak{A})$ by the Fourier multiplier $T_{\varphi}$.

Theorem 5. If $f \in \mathcal{A}_{p}(G, \mathfrak{A})$ then $T_{\varphi} f \in \mathcal{A}_{p}(G, \mathfrak{A})$.

Proof. Let us assume that $f \in \mathcal{A}_{p}(G, \mathfrak{A})$. Then

$$
\begin{aligned}
\left\|\widehat{T_{\varphi} f}\right\|_{\mathcal{L}_{p}}^{p} & =\sum_{\sigma \in \widehat{G}} d_{\sigma} \sum_{i=1}^{d_{\sigma}} \sum_{j=1}^{d_{\sigma}}\left\|\widehat{T_{\varphi} f}(\sigma)\left(\xi_{i}^{\sigma} \otimes \xi_{j}^{\sigma}\right)\right\|^{p} \\
& =\sum_{\sigma \in \widehat{G}} d_{\sigma} \sum_{i=1}^{d_{\sigma}} \sum_{j=1}^{d_{\sigma}}\left\|(\varphi \otimes \widehat{f})(\sigma)\left(\xi_{i}^{\sigma} \otimes \xi_{j}^{\sigma}\right)\right\|^{p} \\
& =\sum_{\sigma \in \widehat{G}} d_{\sigma} \sum_{i=1}^{d_{\sigma}} \sum_{j=1}^{d_{\sigma}}\left\|\varphi(\sigma) \widehat{f}(\sigma)\left(\xi_{i}^{\sigma} \otimes \xi_{j}^{\sigma}\right)\right\|^{p} \\
& \leq \sum_{\sigma \in \widehat{G}} d_{\sigma} \sum_{i=1}^{d_{\sigma}} \sum_{j=1}^{d_{\sigma}}\|\varphi(\sigma)\|^{p}\left\|\widehat{f}(\sigma)\left(\xi_{i}^{\sigma} \otimes \xi_{j}^{\sigma}\right)\right\|^{p} .
\end{aligned}
$$

Since $\varphi$ is bounded, we obtain

$$
\begin{aligned}
\left\|\widehat{T_{\varphi} f}\right\|_{\mathcal{L}_{p}}^{p} & \leq\|\varphi\|_{\infty}^{p} \sum_{\sigma \in \widehat{G}} d_{\sigma} \sum_{i=1}^{d_{\sigma}} \sum_{j=1}^{d_{\sigma}}\left\|\widehat{f}(\sigma)\left(\xi_{i}^{\sigma} \otimes \xi_{j}^{\sigma}\right)\right\|^{p} . \\
& \leq\|\varphi\|_{\infty}^{p}\|\widehat{f}\|_{\mathcal{L}_{p}}^{p}<\infty .
\end{aligned}
$$

Thus $T_{\varphi} f \in \mathcal{A}_{p}(G, \mathfrak{A})$.

Remark 1. From the inclusion property in Theorem 5, One can extend the operator $T_{\varphi}$ to the topological dual $\mathcal{A}_{p}^{*}(G, \mathfrak{A})$ of $\left(\mathcal{A}_{p}(G, \mathfrak{A}),\|\cdot\|_{\mathcal{A}_{p}}\right)$ or $\left(\mathcal{A}_{p}(G, \mathfrak{A}),\|\cdot\|^{\mathcal{A}_{p}}\right)$, exactly as the Fourier tansform is extended from the Schwartz space to the space of tempered distributions, by the relation

$$
\left\langle T_{\varphi} X^{*}, f\right\rangle=\left\langle X^{*}, T_{\varphi} f\right\rangle, X^{*} \in \mathcal{A}_{p}^{*}(G, \mathfrak{A}), f \in \mathcal{A}_{p}(G, \mathfrak{A}) .
$$

Corollary 6. $T_{\varphi}$ is a bounded operator on $\mathcal{A}_{p}(G, \mathfrak{A})$ when the latter is endowed with the norm $\|\cdot\| \mathcal{A}_{p}$.

Proof. In the proof of Theorem 5 we have established that $\left\|\widehat{T_{\varphi} f}\right\|_{\mathcal{L}_{p}} \leq\|\varphi\|_{\infty}\|\widehat{f}\|_{\mathcal{L}_{p}}$. But $\left\|\widehat{T_{\varphi} f}\right\|_{\mathcal{L}_{p}}=\left\|T_{\varphi} f\right\| \mathcal{A}_{p}$ and $\|\widehat{f}\|_{\mathcal{L}_{p}}=\|f\|^{\mathcal{A}_{p}}$. Therefore

$$
\left\|T_{\varphi} f\right\|^{\mathcal{A}_{p}} \leq\|\varphi\|_{\infty}\|f\|^{\mathcal{A}_{p}}
$$

Thus $T_{\varphi}$ is bounded on $\mathcal{A}_{p}(G, \mathfrak{A})$ endowed with the norm $\|.\|^{\mathcal{A}_{p}}$.

Theorem 7. If $T_{\varphi}$ is a bounded operator on $L^{1}(G, \mathfrak{A})$ then $T_{\varphi}$ is also a bounded operator on $\mathcal{A}_{p}(G, \mathfrak{A})$ when the latter is endowed with the norm $\|\cdot\|_{\mathcal{A}_{p}}$.

\section{Proof.}

$$
\begin{aligned}
\left\|T_{\varphi} f\right\|_{\mathcal{A}_{p}} & =\left\|T_{\varphi} f\right\|_{1}+\left\|\widehat{T_{\varphi} f}\right\|_{\mathcal{L}_{p}} \\
& \leq\left\|T_{\varphi}\right\|\|f\|_{1}+\|\varphi\|_{\infty}\|\widehat{f}\|_{\mathcal{L}_{p}} \\
& \leq \max \left\{\left\|T_{\varphi}\right\|,\|\varphi\|_{\infty}\right\}\left(\|f\|_{1}+\|\widehat{f}\|_{\mathcal{L}_{p}}\right) \\
& \leq C\|f\|_{\mathcal{A}_{p}}
\end{aligned}
$$


where $C=\max \left\{\left\|T_{\varphi}\right\|,\|\varphi\|_{\infty}\right\}$. Thus $T_{\varphi}$ is bounded on $\mathcal{A}_{p}(G, \mathfrak{A})$ endowed with the norm $\|\cdot\|_{\mathcal{A}_{p}}$.

Acknowledgments: The authors would like to express their thanks to the referee for his useful remarks.

Author Contributions: All authors contributed equally to the writing of this paper. All authors read and approved the final manuscript.

Conflicts of Interest: "The authors declare no conflict of interest."

\section{References}

[1] Cleanthous, G., Georgiadis, A. G. \& Nielsen, M. (2019). Fourier multipliers on anisotropic mixed-norm spaces of distributions. Mathematica Scandinavica, 124 (2), 289-304.

[2] Julio, D. \& Ruzhansky, M. (2018). Fourier multipliers in Hilbert spaces, in Integral Fourier Operators, Proceedings of a Summer School, ed. Sylvie Paycha and Pierre. J. Clavier, 3 167-191, Germany Universitätsverlag Potsdam, Potsdam.

[3] Rozendaal; J. (2018). Fourier multipliers theorems involving type and cotype. Journal of Fourier Analysis and Applications, 24(2), 583-619.

[4] Atto, E. J., Mensah, Y. \& Assiamoua, V. S. K. (2014). The Fourier multipliers of $p$-Fourier spaces on compact groups. British Journal of Mathematics and Computer Science, 4(5), 667-673.

[5] Mensah, Y. \& Assiamoua, V. S. K. (2010). The $p$-Fourier spaces $\mathcal{A}_{p}(G, A)$ of vector valued functions on compact groups. Advances and Applications in Mathematical Science, 6(1), 59-66.

[6] Assiamoua, V. S. K. \& Olubummo, A. (1989). Fourier-Stieltjes transforms of vector-valued measures on compact groups. Acta Scientiarum Mathematicarum (Szeged), 53, 301-307.

[7] Deitmar, A. \& Echterhoff, S. (2009). Principles of Harmonics Analysis. Springer, New York.

[8] Gaal, S. A. (1973). Linear analysis and representation theory. Springer, Berlin.

[9] Mensah, Y. (2013). Facts about the Fourier-Stieltjes transform of vector measures on compact groups. International Journal of Analysis and Applications, 2(1), 19-25. 